\title{
Preparation and Structure of Microporous Silica Membranes
}

\author{
BALAGOPAL N. NAIR, J.W. ELFERINK, K. KEIZER AND H. VERWEIJ \\ University of Twente, Faculty of Chemical Technology, Laboratory of Inorganic Chemistry and Materials Science, \\ P.O. Box 217, 7500AE Enschede, Netherlands
}

H.Verweij@ct.utwente.nl.

\begin{abstract}
Silica sols have been prepared in an alcoholic solution by hydrolysis and condensation of TEOS (tetraethyl-ortho-silicate) molecules as a function of water and nitric acid concentration. The polymers are intended as precursors for ceramic, gas separation membranes. These molecules show fractal behavior as determined by SAXS (Small Angle X-ray Scattering). Microporosity of dried and calcined silica polymers is determined by $\mathrm{N}_{2}$-adsorption at $77 \mathrm{~K}$. Fractal dimension and porosity increase with increasing acid concentration. Both the sol structure and the drying kinetics determine the porosity values. $\mathrm{N}_{2}$-adsorption isotherms are not very suitable for the determination of pore size distributions of microporous silica.
\end{abstract}

Keywords: polymeric sol, microporous silica, membranes, $\mathrm{N}_{2}$-adsorption, fractals

\section{Introduction}

The development of ceramic membranes has been a research object in our group for a long period [1]. In the book of Scherer and Brinker [2], page 869, such a membrane, prepared in 1987, is shown. The inorganic membranes consist of several porous layers, the thin toplayer being $\gamma-\mathrm{Al}_{2} \mathrm{O}_{3}$ with pores of $2-4 \mathrm{~nm}$. This membrane is suitable for ultrafiltration (perhaps nanofiltration) applications, but the gas separation properties are not very good. These separation properties are based on the Knudsen diffusion mechanism, so separation occurs on basis of the square root of the molar mass ratios of the gas molecules.

To improve gas separation properties, especially for the $\mathrm{H}_{2}$ separation, this standard membrane is modified with an extra top-layer of $\mathrm{SiO}_{2}$ on top or partially inside the $\gamma-\mathrm{Al}_{2} \mathrm{O}_{3}$ layer. The thickness of this layer is about $100 \mathrm{~nm}$ or $1-2 \%$ of the thickness of the $\gamma-\mathrm{Al}_{2} \mathrm{O}_{3}$ layer. Indeed, this membrane has very good separation properties especially for $\mathrm{H}_{2}$ from hydrocarbons. For instance, $\mathrm{H}_{2}$ /propene has a separation factor of 160 [3] and $\mathrm{H}_{2} / \mathrm{CH}_{4}$ of 40 [4], the latter especially at a low level of defects.

The question is now if these types of composite membranes are also suitable for separation of other gas mixtures, for instance by changing the structure of the $\mathrm{SiO}_{2}$ top-layer. Other important gas mixtures to separate are $\mathrm{O}_{2} / \mathrm{N}_{2}, \mathrm{H}_{2} / \mathrm{CO}_{2}$ or hydrocarbon isomers. The structure of the $\mathrm{SiO}_{2}$ top-layer can be changed by:

- changing the size and shape of the 'silica' polymers, so changing the building blocks for the top-layer.

— changing the process parameters during drying and calcination steps.

In this paper we concentrate more on formation of the building blocks, the 'silica' polymers, but some attention will also be paid on drying and calcination steps.

The 'silica' polymers are grown by hydrolysis and condensation reactions starting from tetra-ethyl-orthosilicate (TEOS) as a precursor. The hydrolysis and condensation reactions are shown in the next equations:

$$
\begin{aligned}
& \text { hydrolysis } \rightarrow \\
& \equiv \mathrm{Si}-\mathrm{OR}+\mathrm{H}_{2} \mathrm{O}_{-} \equiv \mathrm{Si}-\mathrm{OH}+\mathrm{ROH} \\
& \quad \leftarrow \text { esterification } \\
& \text { alcohol condensation } \rightarrow \\
& \equiv \mathrm{Si}-\mathrm{OR}+\mathrm{HO}-\mathrm{Si} \equiv{ }_{-} \equiv \mathrm{Si}-\mathrm{O}-\mathrm{Si} \equiv+\mathrm{ROH} \\
& \quad \leftarrow \text { alcoholysis } \\
& \text { water condensation } \rightarrow \\
& \equiv \mathrm{Si}-\mathrm{OH}+\mathrm{HO}-\mathrm{Si} \equiv{ }_{-} \equiv \mathrm{Si}-\mathrm{O}-\mathrm{Si} \equiv+\mathrm{H}_{2} \mathrm{O} \\
& \quad \leftarrow \text { hydrolysis }
\end{aligned}
$$


The reaction rates and the ratio between hydrolysis and condensation reactions depend on the process parameters in the reaction mixture. The main parameters are temperature, TEOS-concentration and the ratios of water to TEOS $\left(r_{w}\right)$, and catalyst to TEOS $\left(r_{a}\right)$. The catalyst can either be protons (acid catalysis) or hydroxyl ions (base catalysis). Protons enhance especially the hydrolysis reaction rate [5], hydroxyl ions especially the condensation reaction rate. Reaction mechanisms can be found in the literature $[2,6]$. Base catalysis enhances condensation and so the formation of particles. If particles are packed, pores will be present between the particles with a size of about $20-40 \%$ of the particle size, depending on the packing. This means that, in most of the cases, pores are larger than $1 \mathrm{~nm}$, resulting in a mesoporous system. This is not the objective of the preparation of gas separation membranes. Therefore protons are chosen as catalysts for a fast hydrolysis and a slow condensation of the hydrolysed species. It should also be emphasized that the reaction rate for hydrolysis of the first OR-group of the TEOS molecule is orders of magnitude larger than that of the second ORgroup [2,6]. The same holds for the third and fourth OR-groups. This means that completely hydrolized TEOS-molecules are almost not present.

Condensation leads in most cases to fractal objects, which can be represented with a mass fractal dimension $D_{f}$, related to mass $M$ and size $R$ of polymeric molecule $\left(M \sim R^{D_{f}}\right)$. $D_{f}$ has a limiting value of three, where the object is euclidean. As the fractal dimension decreases, the structure will look sparser, giving essentially a linear configuration at $D_{f}=1$. This is the case for polymeric molecules obtained through condensation of single-hydrolysed TEOS molecules. The tendency of fractal systems to interpenetrate for instance in the drying process, is inversely related to the mean number of intersections [6]. Smaller fractal dimensions encourage interprenetation [7]. Fractal dimension $D_{f}$ and size $R_{g}$ (radius of gyration) will therefore be used as characterization parameters of the polymeric sol species.

Porosity and pore size of the dried and calcined 'silica' membrane material will be characterized with $\mathrm{N}_{2}$ adsorption at $77 \mathrm{~K}$. The pore size is calculated with a Horvath-Kawazoe model [8].

\section{Experiments and Results}

Within this investigation, the parameters $r_{a}$ and $r_{w}$ are chosen to vary. This is because these parameters are

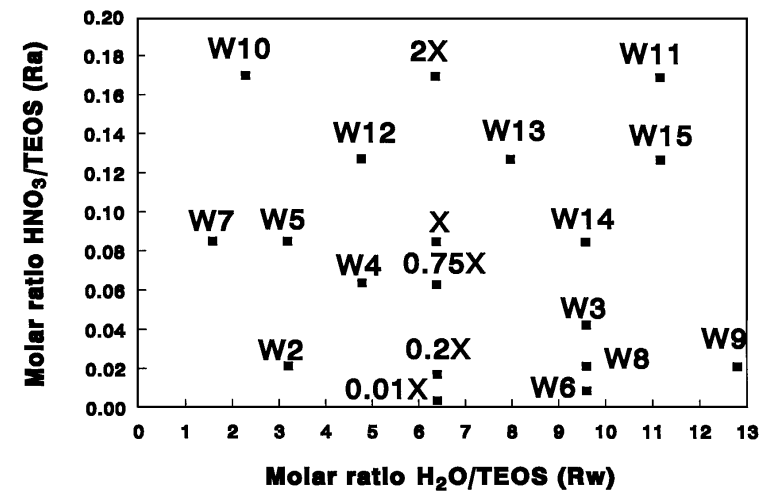

Figure 1. Composition of sols with corresponding sol-codes.

most important for controlling size and shape of the 'silica' molecules. The other important parameters are chosen constant at values which make [TEOS]concentration $2 \mathrm{M}$; synthesis temperature is $65^{\circ} \mathrm{C}$ and synthesis time is $3 \mathrm{hrs}$. Before performing SAXS analysis $\left(D_{f}\right.$ and $\left.R_{g}\right)$, the samples are kept at $-20^{\circ} \mathrm{C}$ in a freezer, because the sample mixture is still reactive. More details are given by de Lange et al. [6, 9], Nair et al. [10] and Elferink et al. [11].

The standard reaction composition for preparation of silica polymers is $\mathrm{X}[3,9]$, as shown in Fig. 1. Starting from these values, $r_{a}$ and $r_{w}$ are varied within practical limits. Too large values of either $r_{a}$ or $r_{w}$ result in gelling or in mesoporous structures. Under the mentioned process conditions, the limiting values for $r_{a}$ and $r_{w}$ are about 0.2 and 12 , respectively.

In Fig. 2, a typical SAXS-curve is shown in which the $\log$ of scattering intensity $I$ is given as a function of the log of the scattering vector, where the scattering

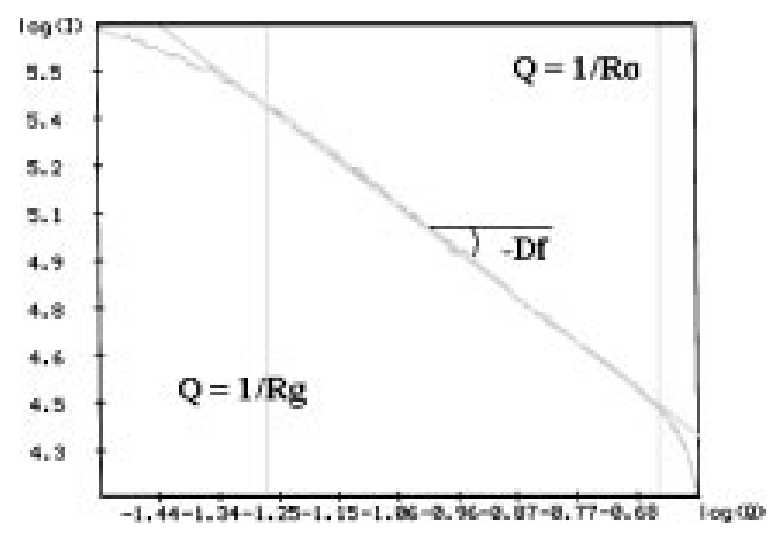

Figure 2. SAXS-diagram, with $\log$ (Intensity) as a function of $\log \left(Q\right.$, scattering vector). Determination of $R_{g}, D_{f}$ and $R_{0}$ are indicated. 


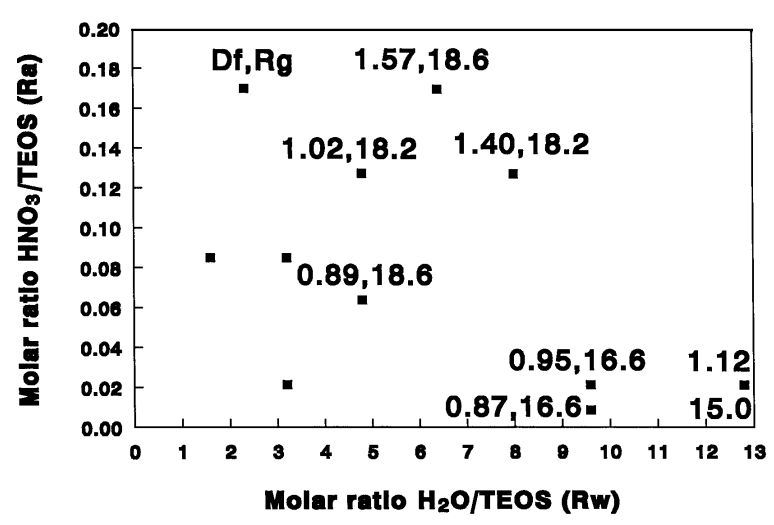

Figure 3. Radius of gyration $\left(R_{g}\right)$ and fractal dimension $\left(D_{f}\right)$ for some sol compositions.

angle $\Theta$ is incorporated $(Q=2 \pi / \lambda \sin (2 \Theta)$ with $\lambda$ is wave length of radiation). From these curves, $R_{g}$ and $D_{f}$ can be determined ( $R_{0}$ represents the size of the 'silica-monomer').

In Fig. 3, some values of $D_{f}$ and $R_{g}$ are shown for the standard process conditions. At low $r_{w}\left(r_{w}<4\right)$ and $r_{a}$ $\left(r_{a}<0.02\right)$, the $D_{f}$-values determined from the SAXScurves are smaller than 1 (not all shown in Fig. 3). No physical model is valid for such low $D_{f}$-values, but it is more often found for reactive systems with growing structures [12]. For $r_{w}>4, D_{f}$ increases with increasing values of $r_{a}$ and $r_{w}$. The radius of gyration $R_{g}$ is, under the chosen process parameters, almost independent of $r_{a}$ and $r_{w}$ and has a value of 15-20 $\AA$.

It appears, that $3 \mathrm{hrs}$ of reaction time at $65^{\circ} \mathrm{C}$ is rather short for obtaining larger polymers with fractal dimensions larger than 1. Only for high $r_{a}$ values $\left(r_{a}>0.1\right)$ and $r_{w}$ values $\left(r_{w}>4\right)$, more branching occurs. Long time experiments (up to about 10,000 hrs [10]) at room temperature showed that growing of particles still occurs. At 1,000 hrs, the $D_{f}$ and $R_{g}$ values of sample X (Fig. 1) are 1.8 and $35 \AA$, respectively.

After synthesis time of $3 \mathrm{hrs}$, the reaction mixtures are dried under ambient conditions (room temperature, about $60 \% \mathrm{RH}$ ) in a PE petri dish. Here it should again be emphasized that the reaction does not stop at room temperature [10] but it is much slower than at $60^{\circ} \mathrm{C}$. However, during drying, concentrations increase and hydrolysis and condensation reaction rates increase. Therefore, drying conditions and size of the polymeric sol samples are very important. The latter is very different for the samples prepared for this investigation (unsupported) and the supported 'silica' samples. The thickness of the silica membrane top layer is about 1,000 smaller than the thickness of 'unsupported'

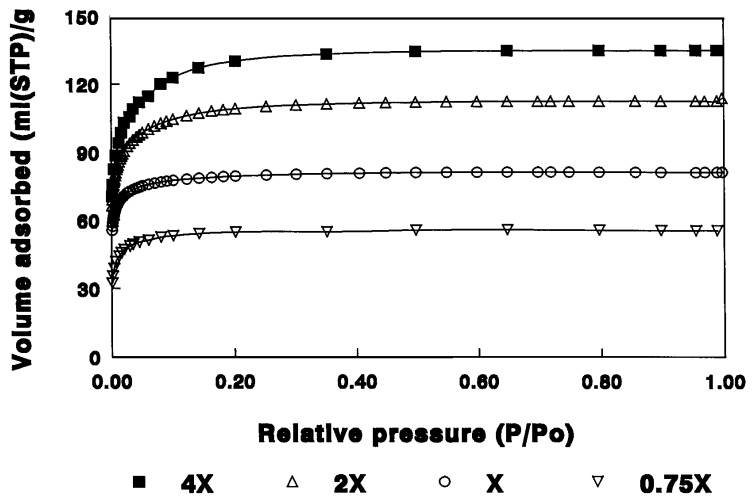

Figure 4. $\mathrm{N}_{2}$-adsorption isotherms as a function of $r_{a}$ at a constant value of $r_{w}(=6.4)$ (see also Fig. 2).

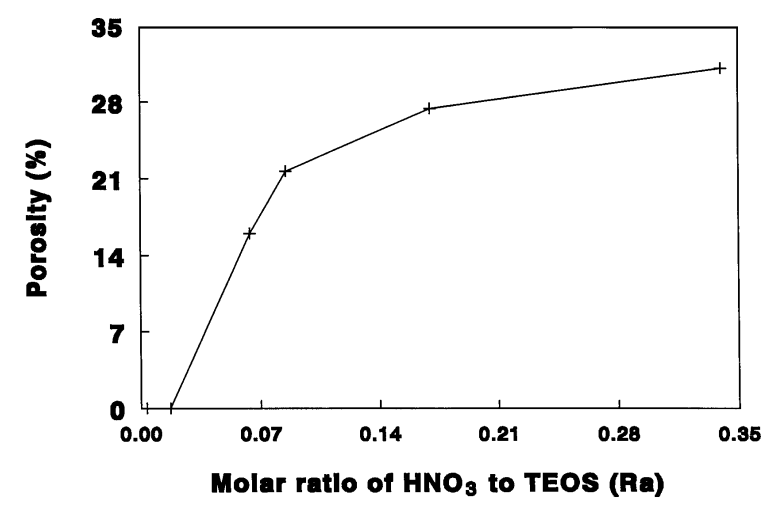

Figure 5. Porosity of calcined samples as a function of $r_{a}$ (see Fig. 5).

membranes. Drying time is then a matter of seconds for supported samples and hours for the samples used in this investigation.

After drying, the samples are heat-treated at $400^{\circ} \mathrm{C}$ during 3 hrs. In Fig. $4 \mathrm{~N}_{2}$-adsorption, measured at 77 $\mathrm{K}$, is shown as a function of the relative pressure of $\mathrm{N}_{2}$ for samples with different $r_{a}$-values and a $r_{w}$-value of 6.4. From these curves, the porosity accessible to $\mathrm{N}_{2}$ can be calculated and is shown in Fig. 5. It is clear that porosity increases with increasing value of $r_{a}$. Porosity changes with $r_{w}$ are not so clear, but there is a tendency, for $r_{w}>4$, that porosity increases with increasing $r_{w}$. For the low $r_{w}$ values, the porosity is surprisingly large, of the order of that of sample 4X (Fig. 4). For low $r_{a}$ values, the calcined materials are $\mathrm{N}_{2}$-dense.

The shape of all adsorption curves, especially the steepness of increase of adsorption with relative pressure, at low $\mathrm{N}_{2}$-pressures, is the same. Because pore sizes are calculated from this part of the adsorption 
curve (with all types of models), the pore size distribution is also the same for all samples. Calculation of pore sizes with a modified Horvath-Kawazoe model showed a pore size distribution with a maximum at $5 \AA$ and a small secondary maximum at $7.5 \AA$. It is possible that, in other models, an absolute difference of 1-2 in pore size values can be found.

\section{Discussion}

This discussion is a starting point for some of the phenomena which occur in dynamic systems of growing ' $\mathrm{SiO}_{2}$ ' polymers in organic liquids and the following consolidation steps. It is rather speculative and is meant to open the discussion about phenomena, which are important for practical applications of sol-gel technology, but are often avoided in scientific considerations.

Three subjects will be treated; low fractal dimensions, microporosity formation in consolidated structures and $\mathrm{N}_{2}$-adsorption for pore size analysis in microporous materials.

\section{Low Fractal Dimensions}

It is difficult to ascribe a physical model for $D_{f}$ values smaller than 1, which are determined from SAXScurves. According to Martin and Hurd [12], the lowest possible $D_{f}$ for a connected fractal is one. In his Ph.D. thesis, Dokter [13] presented a mass fractal dimension curve as a function of time, in a ' $\mathrm{SiO}_{2}$ ' growing system, starting from 0.8 . These references and our own results show that $D_{f}$ values smaller than 1 exist in practical systems, termed here as disconnected fractals. Hydrolyzed species are formed, but condensation did not start to form the polymers. The hydrolyzed species are ordered in a certain way (like micelles) into domains with about $15 \AA$ radius. Density differences occur in the system, which can lead to a certain 'apparent' fractal behaviour.

\section{Porosity}

The relation between sol properties and resulting porosity of the dried and calcined material is somewhat confusing. The following discussion gives a possible explanation for the observed results.

For $r_{w}>4$, the acid concentration controls the hydrolysis and condensation rates. The hydrolysis rate is largest, but also some condensation takes place. In the reaction mixture, a dynamic equilibrium structure is present between partially-hydrolyzed monomers and polymers formed by condensation. During drying, the concentrations of reactants and catalyst are increased. Therefore, hydrolysis and condensation reaction rates are also increased and new interconnections between polymers or polymers and monomers are formed. This prevents interpenetration, occurring at $D_{f}=1.5$ [6], of the polymers and a higher porosity is present after drying and calcinating (Fig. 4/5). If the catalyst concentration is low, almost no polymers are formed and also during drying the condensation rate is slow. This means packing of (partially-hydrolyzed) monomers and packing can be dense (Fig. 5).

At low $r_{w}$, the situation is even more confusing. Of course, the reaction rates for hydrolysis and condensation is slower than at higher water content. This $r_{w}$ value is smaller than the stoichiometric amount necessary for the hydrolysis reaction 1 . Because of equilibrium between hydrolysis and condensation (reactions 1-3), the water condensation reaction becomes relatively more important because of generation of water. Maybe that, in the reaction mixture, a large concentration of monomer and a small concentration of relatively large and branched polymers is present, because the latter generate the water. It is then not clear how the SAXS results and determined $D_{f}$-values should be interpreted and the question about a fractal system is also not clear. During drying, condensation becomes even more important. Interpenetration of polymers is difficult (perhaps $D_{f} \cdot 1.5$ ) and porosity of dried and calcined samples is relatively high.

The previous discussion is speculative, but it is an attempt to interpret phenomena, which occur during reaction and drying process. With this discussion, it is clear that the sol structure obtained after $3 \mathrm{hrs}$ of synthesis is not the only factor which determines the structure after drying and calcination. The ratio between drying rate and condensation rate during drying can be just as important for the consolidated structure. Lange et al. [6] found also that faster drying rates (large drying/condensation ratio) leads to denser consolidated structures. From this discussion, it has to be concluded that structure differences between 'unsupported' membranes as treated in this paper and 'supported' membranes, used for applications, occur. Drying of supported membranes is much faster and the structure is then much denser. This can explain the good $\mathrm{H}_{2}$-separation properties of these types of membranes. 


\section{$\mathrm{N}_{2}$-Adsorption}

$\mathrm{N}_{2}$-adsorption experiments always gave nearly the same pore size distribution for the calcined silica samples. The question arises if this method is a good static characterization technique for amorphous, 100\%microporous material. In our opinion, the answer for this question is no. Firstly, $\mathrm{N}_{2}$ is too large a molecule (3.6 $\AA$ ). Part of the pores are not accessible for $\mathrm{N}_{2}$. The smallest molecules available are $\mathrm{H}_{2}$ and $\mathrm{He} . \mathrm{H}_{2}$ adsorbs in rather large amounts at $77 \mathrm{~K}$ (about $40 \%$ of the amount of $\mathrm{N}_{2}$ in $\mathrm{ml} \mathrm{STP/g}$ at $100 \mathrm{kPa}$ ). No model is available for interpretation of this adsorption isotherm in terms of pore size, because $77 \mathrm{~K}$ is much larger than the critical temperature of $\mathrm{H}_{2}(33 \mathrm{~K})$ and no critical pressure $P_{0}$ is then available for the models. Another possibility is $\mathrm{CO}_{2}$-adsorption at $195 \mathrm{~K}$. According to Sharp [14], $\mathrm{CO}_{2}$-adsorption is larger and faster in these types of microporous materials because of higher adsorption temperature and slightly smaller molecular size. No attempts have been carried out yet to translate $\mathrm{CO}_{2}$-adsorption to pore size distribution. A problem is that $\mathrm{CO}_{2}$ can adsorb preferentially on polar sites.

Secondly, it is possible that the amorphous porous silica structure cannot be characterized by adsorption at all. Perhaps adsorption sites are available with a distribution in adsorption energies, which are not directly related to pore size. One should imagine that sizes near $3 \AA$ are discussed, which is of the order of atomic (ionic) distances in crystal lattices. The philosophical question arises then at which size does the concept of micropores stop and the concept of dense materials starts. At this moment, we still accept the concept of $\mathrm{N}_{2}$-dense at $77 \mathrm{~K}$, because no other standard characterization methods are yet available. However, in future research of these types of materials, other concepts should be developed and other characterization techniques should be available. Another interpretation of SAXS results is perhaps the possibility to calculate pore sizes for these types of microporous silica materials, but there are no examples in literature available yet.

\section{Conclusions}

For the preparation of inorganic membranes with gas separation properties, a microporous or dense structure is a necessity. To build this structure, polymeric silica sols are used with fractal dimensions smaller than 1.5, to obtain interpenetration of the molecules. The silica polymers are obtained by reaction of TEOS with water, in the presence of nitric acid (protons) as a catalyst.
The $r_{w}$ (water/TEOS ratio) and $r_{a}$ (acid/TEOS-ratio) are varied between 0 and 12 and 0 and 0.35 respectively. Fractal dimension $D_{f}$ increases with increasing $r_{a}$ value. $D_{f}$ is sometimes below 1 , which has no physical meaning in fractal theory. It is explained as the presence of hydrolyzed molecules ordered in very small domains ( $15 \AA$ ), which are not yet connected by condensation. One can speak of an 'apparent' fractal dimension.

The polymer solution is dried and calcined at $400^{\circ} \mathrm{C}$. A microporous structure is obtained, whose porosity increases with increasing $r_{a}$. This is explained in terms of acceleration of the condensation reaction rate during the drying process. Interpenetration then becomes difficult. Porosity is also large for systems prepared with water concentrations less than the value for complete hydrolysis $\left(r_{w}<4\right)$. Also, in that case, condensation during drying may have the main role.

A final remark is that $\mathrm{N}_{2}$-adsorption is perhaps not a good technique for the determination of pore size distribution of small micropores, as found for these materials. For these types of materials, new characterization techniques should be developed.

\section{References}

1. A.F.M. Leenaars and A.J. Burggraaf, J. Colloid Interface Science 105, 27 (1985).

2. C.J. Brinker and G.W. Scherer, Sol-Gel Science: The Physics and Chemistry of Sol-Gel Processing (Academic Press, San Diego, 1990), Chap. 3.

3. R.J.R. Uhlhorn, K. Keizer, and A.J. Burggraaf, J. Membrane Science 66, 271 (1992).

4. R.S.A. de Lange, K. Keizer, and A.J. Burggraaf, I\&EC Research (1995) accepted.

5. R. Aelion, A. Loebel, and F. Eirich, J. Amer. Chem. Soc. 72, 5705 (1950).

6. R.S.A. de Lange, J.H.A. Hekkink, K. Keizer, and A.J. Burggraaf, J. Non-Cryst. Solids (1995), accepted.

7. C.J. Brinker, R. Sehgal, S.L. Hietala, R. Deshpande, D.M. Smith, D. Loy, and C.S.S. Ashley, J. Membrane Science 94, 85 (1994).

8. R.S.A. de Lange, K. Keizer, and A.J. Burggraaf, J. Porous Materials 1, 139 (1995).

9. R.S.A. de Lange, K.-N.P. Kumar, J.H.A. Hekkink, G.M.H. van de Velde, K. Keizer, A.J. Burggraaf, W.H. Dokter, H.F. van Garderen, and T.P.M. Beelen, J. Sol-Gel Science and Techn. 2, 489 (1994).

10. B.-N. Nair, W.E. Elferink, K. Keizer, and A.J. Burggraaf, J. Colloid Interface Science (1995), accepted.

11. W.E. Elferink, B.-N. Nair, R.M. de Vos, K. Keizer, and H. Verweij, J. Colloid Interface Science (1995), submitted.

12. J.E. Martin and A.J. Hurd, J. Appl. Cryst. 20, 61 (1987).

13. W.H. Dokter, Transformation in Silica Gels and Zeolite Precursors (Ph.D. Thesis, Technical University of Eindhoven, 1994), p. 61.

14. K.G. Sharp, J. Sol-Gel Science and Technology 2, 35 (1994). 